\title{
Diagnostic Yield of Capsule Endoscopy in Gastroduodenal and Small Bowel Disease: An Experience from a Local Hospital in Gaza Strip: Experience, Comparison to Other Existing Modalities and Utility as Capsule Gastroduodenoscopy
}

\author{
Matar K $^{1^{*}}$, Helal A ${ }^{1}$, Al Shami Salah ${ }^{1}$, Ashour Mohammed ${ }^{2}$ and Abo Jazr Iyad ${ }^{1}$ \\ ${ }^{1 *}$ European Gaza hospital-Gaza Strip- Palestine, Israel \\ ${ }^{2 *}$ Public Aid Hospital-Gaza Strip-Palestine, Israel
}

Received: March 09, 2018; Accepted: March 29, 2018; Published: April 09, 2018

*Corresponding author: KhaledMatar, European Gaza hospital, Gastroenterology department, Khan Younis City-Alfukhari, Gaza Strip-Palestine, Tel ; 0592155123; E-mail: aboayman2001@hotmail.com

\begin{abstract}
Capsule endoscopy (CE) is a safe, novel technology that allows direct noninvasive visualization of the entire small intestine. CE permits a detailed examination in the ambulatory setting, allowing identification of clinically relevant lesions, and it is appealing to both patients and providers. Video capsule endoscopy (VCE) is a powerful diagnostic tool that has proved especially useful in imaging the small intestine. In this study we will review its efficacy in as a diagnostic utility for Gastroduodenal lesions as a capsule Gastroduodenoscopy.
\end{abstract}

Keywords: Capsule Endoscopy; Crohns disease; Small Bowel;

\section{Methods}

We carried out a retrospective audit of all patients referred for CE in our hospital between 2015 and 2017 with suspected small bowel or gastroduodenal pathology undetected or missed by conventional means to evaluate the diagnostic yield of CE and its impact on patients' management.

\section{Results}

Small bowel pathology was suspected in patients with symptoms of either abdominal pain or diarrhea and weight loss, iron deficiency anemia (IDA), or obscure overt or occult upper gastrointestinal bleeding. Almost all patients presenting with these symptoms had small bowel radiology before proceeding to CE which was unrevealing.

40 patients underwent CE during the study period. 10 (25\%) patients were females. A diagnosis of Crohn's disease was made in 7 (17.5\%) patients on CE based on the findings of erosions, aphthous ulcers etc. $13(32.5 \%)$ patients had a normal examination, 1 (2.5\%) had NSAID induced ulcers, 2 (5 $\%)$ were diagnosed with small bowel tumors, 10 (25\%) had erosive gastropathy, gastric and duodenal ulcers, 5 (12.5\%) were diagnosed with small bowel angiodysplasia, and 2 (5\%) had limited views due to poor bowel preparation for which a repeat CE examination was requested.

CE changed management in 19 (47.5\%) patients and included the following: 7 patients with Crohn's disease were managed conservatively, NSAIDs were stopped in one patient, proton pump inhibitor and helicobacter pylori eradication therapy was started in 10 patients, one patient had gold probe cauterization for colonic and gastric angiodysplasia.

\section{Conclusion}

In our selected cohort, small bowel Crohn's disease was diagnosed in $17.5 \%$ of patients that was undetectable by conventional means. This figure is lower than that mentioned in the literature (43-71\%). Other significant diagnoses were obtained in $45 \%$ of cases (NSAID induced ulcers, small bowel angiodysplasia, and gastroduodenal ulcers and erosive gastropathy) and were managed conservatively and endoscopically with good outcome. Small bowel tumors were diagnosed in 5\%. We recommend that all patients should have the appropriate investigations done prior to $\mathrm{CE}$ as opposed to CE being a first line investigation. CE is a very useful investigative tool to view not only the small bowel pathology but also in certain circumstances as capsule gastroduodenoscopy, as it has a good diagnostic yield in indentifying lesions in the stomach, duodenum and small bowel when the other investigations are unyielding. Identifies as many upper GI pathologies as OGD in addition to small bowel pathologies CE may be suitable for gastric screening compared with gastroduodenoscopyscopy, and can be used to screen gastric diseases without sedation. 


\section{References}

1. Robert Enns. Capsule Endoscopy in Patients with Iron Deficiency. Gastroenterol Hepatol (N Y). 2012;8(12):847-849.

2. Fireman Z, Eliakim R, Adler S, Scapa E. Capsule endoscopy in real life: a four-centre experience of 160 consecutive patients in Israel. Eur J Gastroenterol Hepatol. 2004;16(9):927-931.

3. Rodrigues-Pinto E, Cardoso H, Rosa B, Santos-Antunes J, Rodrigues S Marques M, et al. Development of a predictive model of Crohn's disease proximal small bowel involvement in capsule endoscopy evaluation. Endosc Int Open. 2016;4(6):E631-6. doi: 10.1055/s-0042-106961

4. Kopylov U, Yung DE, Engel T, Vijayan S, Har-Noy O, Katz L, et al Diagnostic yield of capsule endoscopy versus magnetic resonance enterography and small bowel contrast ultrasound in the evaluation of small bowel Crohn's disease: Systematic review and meta-analysis. Dig Liver Dis. 2017;49(8):854-863. doi: 10.1016/j.dld.2017.04.013
5. Kobayashi Y, Watabe H, Yamada A, Hirata Y, Yamaji Y, Yoshida H, et al. Diagnostic yield of capsule endoscopy for gastric diseases. Abdom Imaging. 2012;37(1):29-34. doi: 10.1007/s00261-011-9777-y

6. Carlos Robles-Medranda, Hannah P Lukashok, Raquel S Del Valle, Cesar Mariscal, Miguel A Chung Sang, Gustavo Rubio, et al. Tu1558 Capsule Endoscopy for Detection of Gastritis and Gastric Lesions in Dyspeptic Patients: A Pilot Study. 2011;73(4): AB446-AB447.

7. Liao Z, Hou X, Lin-Hu EQ, Sheng JQ, Ge ZZ, Jiang B, et al. Accuracy of Magnetically Controlled Capsule Endoscopy, Compared With Conventional Gastroscopy, in Detection of Gastric Diseases. Clin Gastroenterol Hepatol. 2016;14(9):1266-1273.e1. doi: 10.1016/j. cgh.2016.05.013

8. H-L Ching, MF Hale, JA Campbell, A Healy, V Thurston, R Sidhu, et al. PTU-019 Magnetically steered capsule endoscopy (msce) of the upper and mid gut in recurrent and refractory iron deficiency anaemia. $2017 ; 66(2)$ 